\title{
Resolving the jet in Cygnus A
}

\author{
U. Bach ${ }^{* a}$, T. P. Krichbaum ${ }^{a}$, E. Middelberg ${ }^{b}$, W. Alef ${ }^{a}$, and J. A. Zensus \\ ${ }^{a}$ Max-Planck-Institut für Radioastronomie, Bonn, Germany \\ ${ }^{b}$ Astronomisches Institut - Ruhr Unsiversität, Bochum, Germany \\ E-mail: ubach@mpifr-bonn.mpg.de, tkrichbaum@mpifr.de, \\ middelberg@astro.rub.de, walef@mpifr.de, and azensus@mpifr.de
}

Cygnus A is the closest $(z=0.057)$ strong FR II radio galaxy and therefore a key object for detailed studies of its prominent double sided jet and nucleus. Owing to the large inclination of the jet with respect to the observer $\left(>75^{\circ}\right)$, and correspondingly reduced relativistic effects which allow to measure directly the jet speed, Cyg A is an ideal candidate for detailed studies of its jet physics, which is thought to be similar to those in the more luminous quasars. Our previous studies revealed a good kinematic model for the jet of Cygnus A, but the counter-jet speed is still not well constrained. The central engine and part of the counter-jet of Cyg A are likely to be obscured by free-free absorbing material, presumably a thick torus. At mm-wavelengths, the absorber becomes optically thin, which provides a more detailed view into the inner nuclear region. Knowing the speed of jet and counter-jet and their flux density ratio allows to determine the jet Lorentz factors and orientation. Therefore we started to monitor Cyg A with global VLBI at $43 \mathrm{GHz}$ in Oct. 2007. Our first epoch reveals a previously unseen gap between both jets. This could be either a sign for a new counter-jet component that is slowly separating or we start to see the very inner acceleration region of the jet which is not efficiently radiating at radio wavelengths. Further more the image shows transversely resolved jet structures at distances beyond $\sim 0.5 \mathrm{pc}$ which facilitate more detailed investigations addressing jet stratification. Analysis of the resolved jet structure shows that the initially wide jet (opening angle $\sim 10^{\circ}$ ) collimates within the first parsec into a edge-brightened jet with an opening angle of $\sim 3^{\circ}$.

The 9th European VLBI Network Symposium on The role of VLBI in the Golden Age for Radio Astronomy and EVN Users Meeting

September 23-26, 2008

Bologna, Italy

\footnotetext{
* Speaker.
} 


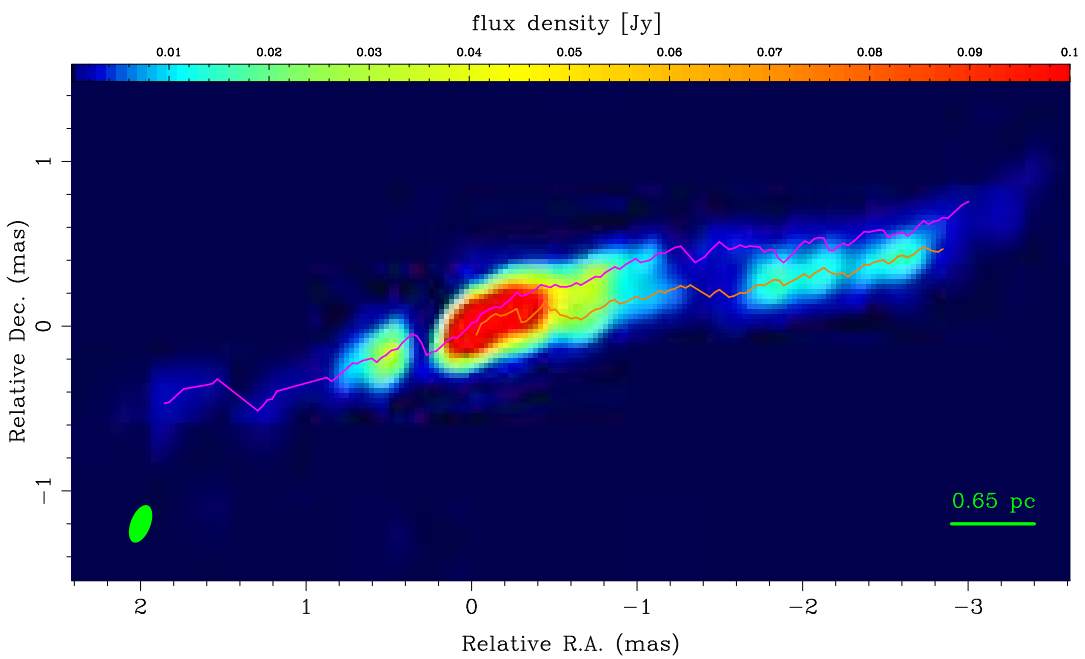

Figure 1: Global 7mm VLBI image from Oct. 2007 (GB060). The image clearly shows that the jet is transversely resolved and for some part of it, two ridge-lines along the edges of the jet can be fitted (overlaid lines in pink and orange, see text for details). With a peak flux density of $180 \mathrm{mJy}$ and an off-source rms noise of $0.2 \mathrm{mJy}$ the dynamic range is about 900 to 1 . Noticeable is also the very sharp emission gap at around 0.2 mas, which separates the jet from the counter-jet. The nature of the gap is not clear yet, but could well mark the location of the central engine.

Observations To obtain the highest possible sensitivity and resolution a global VLBI array of 15 antennas, including the VLBA, GBT, VLA1, Effelsberg, Onsala, and Noto, at $43 \mathrm{GHz}$ with a recording rate of $512 \mathrm{Mbps}$ is used to image Cygnus A on four successive epochs separated by about 8 month. The first epoch was observed in October 2007, which will be followed by observations in October 2008, February 2009, and October 2009. The data from the first epoch was correlated at JIVE. Standard data reduction was applied in AIPS, imaging and model fitting was done in Difmap. Except for the non detection of fringes to Noto due to a fault of a synthesizer, all stations worked well and the a priori calibration was good within $10 \%$ to $20 \%$ for most of the time.

Results and Analysis As seen in Fig. 1 the jet is transversely resolved at separations of more than 0.2 mas from the VLBI core. To measure the width of the jet the task SLICES in AIPS was used to obtain transverse flux density profiles of the jet and the counter-jet. Slices were taken at every pixel ( 0.03 mas) from 2.5 mas to -3.5 mas relative RA. Some example profiles are shown in the left panel of Fig. 2. The first image (top, left panel) at $r=0.0$ mas shows the still compact inner jet, but with larger separations from the core the jet broadens and shows a limb-brightened structure (top right, bottom left to right). To parameterize the flux density profiles two Gaussian components were fitted to the data. The core and the very inner jet are well represented by a single Gaussian. At about 0.3 mas to 0.5 mas from the core the profiles are better represented by two Gaussian and both are slowly separating from each other with increasing distance from the core. The two ridge-lines show in Fig. 1 correspond to the peak positions of the Gaussian fits and follow nicely the bright rims of the jet. For comparison all profiles were also fitted with a single Gaussian. The flux density profile along the jet and the jet width derived from the single Gaussian fit against core separation are shown in Fig. 2 (right panel). Remarkable in the flux density profile is the sharp emission gap that seems to separate the jet from the counter-jet. It is also the region were the jet 

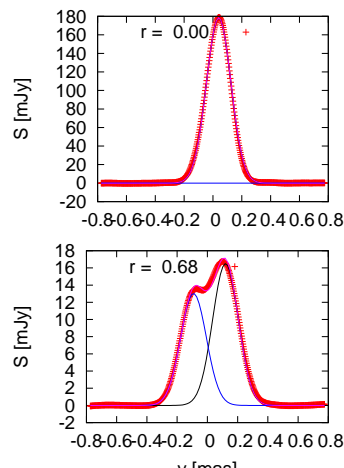

$\mathrm{y}[\mathrm{mas}]$
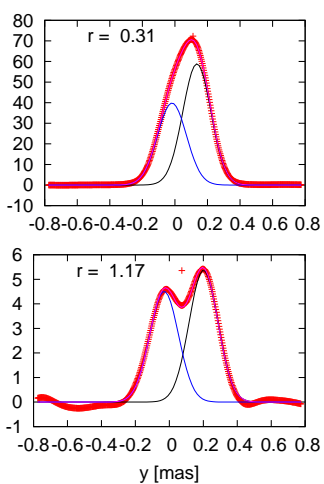

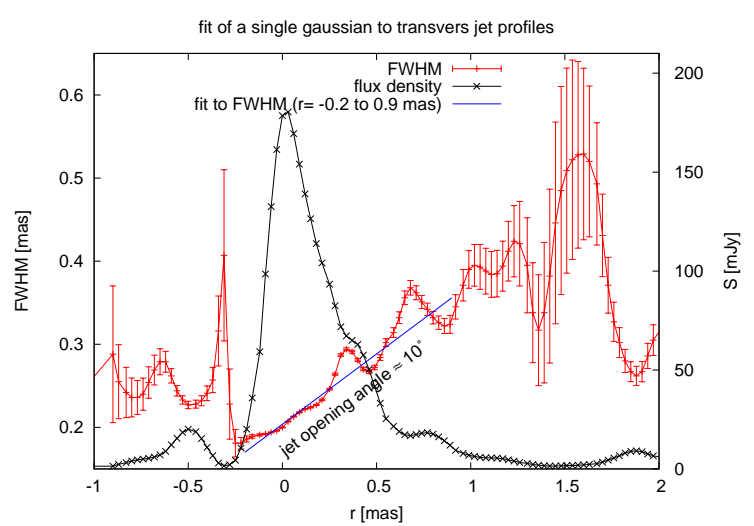

Figure 2: Left: Flux density profiles taken transversely to the jet direction. The jet is clearly resolved at distances larger than 0.5 mas away from the VLBI core. To parameterize the flux density profiles two Gaussian were fitted to the data (blue and black line, pink is the sum of both). Right: The jet opening angle is obtained from the width of the Gaussian fit to the transverse profiles. For comparison the flux density profile along the jet axis is shown (scale on right axis).

is most compact. The nature of the gap is not clear yet. Possibilities include strong absorption by the inner torus/accretion-disc or that it is the region where the jets are formed and where they are less radiative. The jet width derived from the analysis of the jet ridge-line shows a strong increase in the inner jet ( $<0.8$ mas) that corresponds to a jet opening angle of $\sim 10^{\circ}$.

The opening angle of the more extended jet can be derived from the increasing separation of the northern and southern ridge-lines (Fig. 1). Here we obtain an angle of only $2-3^{\circ}$. This suggests that we start to resolve the initial jet collimation region. Interestingly the collimation appears in the inner $1.5 \mathrm{pc}$ in agreement to the region where the jet seems to be accelerated [1]. A similar structure is observed also in M87 [2, 3]. The further analysis, specially the linear polarization images, the following epochs, and the comparison to global $3 \mathrm{~mm}$ images [4] should show if e.g. the gap really marks the position of the central engine and if we can see here into the region where jets are formed and accelerated [5, 6]. However, without question, this observations should facilitate more detailed investigations and allow a better comparison to MHD simulations like those recently done for e.g. M87, 3C 273 [7], and 0836+714 [8].

\section{References}

[1] Bach, U., et al. 2005, in ASP Conf. Ser. 340, ed. J. D. Romney \& M. J. Reid, 30

[2] Junor, W., Biretta, J. A., \& Livio, M. 1999, Nature, 401, 891

[3] Kovalev, Y. Y., Lister, M. L., Homan, D. C., \& Kellermann, K. I. 2007, ApJ, 668, L27

[4] Krichbaum, T. P., 2008, Proc. of the 9th EVN Symposium, PoS (IX EVN Symposium) 001

[5] Vlahakis, N. \& Königl, A. 2004, ApJ, 605, 656

[6] Marscher, A. P., Jorstad, S. G., D’Arcangelo, F. D., et al. 2008, Nature, 452, 966

[7] Lobanov, A. P. \& Zensus, J. A. 2001, Science, 294, 128

[8] Perucho, M. \& Lobanov, A. P. 2007, A\&A, 469, L23 